\title{
On the Steering of Sound Energy Through a Supercritical Plate by a Near-Field Transducer Array
}

Brian E. Anderson

Stephen A. Hambric

Jack W. Hughes

Follow this and additional works at: https://scholarsarchive.byu.edu/facpub

Part of the Astrophysics and Astronomy Commons, and the Physics Commons

\section{Original Publication Citation}

B. E. Anderson, W. J. Hughes, and S. A. Hambric, "On the steering of sound energy through a supercritical plate by a near-field transducer array," J. Acoust. Soc. Am., 124, 2613-2619 (28).

\section{BYU ScholarsArchive Citation}

Anderson, Brian E.; Hambric, Stephen A.; and Hughes, Jack W., "On the Steering of Sound Energy Through a Supercritical Plate by a Near-Field Transducer Array" (2008). Faculty Publications. 897.

https://scholarsarchive.byu.edu/facpub/897 


\title{
On the steering of sound energy through a supercritical plate by a near-field transducer array
}

\author{
Brian E. Anderson, ${ }^{\text {a) }}$ W. Jack Hughes, and Stephen A. Hambric \\ Applied Research Laboratory, The Pennsylvania State University, P.O. Box 30, State College, \\ Pennsylvania 16804, USA
}

(Received 19 October 2007; revised 13 December 2007; accepted 7 February 2008)

\begin{abstract}
The ability to direct sound energy through the flexural vibrations of a submerged plate at various angles of incidence using a near-field transducer array is investigated. An alumina bar is placed in front of a one-dimensional, eight-element transducer array, between the array and the water. Operating in a receive mode, data were taken as a function of angle of incidence and compared to data taken without the presence of the alumina bar. The array was also operated in transmit mode and results were compared to corresponding receive mode data, showing that reciprocity holds. Results show that in fact sound energy can be steered through a plate, and that the measurement method used provides a convenient method of measuring the angular dependence of transmission through a plate, including measurements at frequencies above the plate's critical frequency. Experimental results of sound transmission versus angle of incidence of finite sized plates agree qualitatively with theoretical results from an analysis of the transmission through an unbounded flexible partition. (C) 2008 Acoustical Society of America. [DOI: 10.1121/1.2890738]
\end{abstract}

PACS number(s): 43.55.Rg, 43.38.Hz, 43.30.Yj, 43.20.Ye [DF] Pages: 2613-2619

\section{INTRODUCTION}

Arrays of transducer elements are often used to channel sound radiation into specific directivity patterns to image objects at specific angles. In some applications it is desirable to send sound through a plate or wall to allow imaging of objects on the other side, especially when it is not feasible to physically inspect the other side of the plate or wall. One potential application would be to place an array on the inner surface of the hull of a ship or submarine. There have been various studies on the effects of steering an array through acoustic windows, including modeling and testing, ${ }^{1-7}$ but the main goal of those studies is to design an acoustically transparent barrier between the array electronics and the water. This paper investigates the steering of sound energy through a plate with an array mounted to the plate and proposes a method to measure the angular dependence of sound transmission through a plate.

The critical or coincidence frequency for sound transmission is the frequency at which the speed of sound in the acoustic fluid matches that of the speed of bending waves in a plate, resulting in nearly perfect transmission of sound at grazing incidence. As frequency is increased, the angle at which the coincidence effect occurs (high degree of sound transmission) shifts progressively away from grazing incidence and closer to normal incidence. The angular shifting of the coincidence effect occurs due to the dispersive nature of bending waves in plates. The theory of the coincidence effect and its frequency dependence is generally credited to Cremer. ${ }^{8}$ Cremer's work in 1942 has influenced many other researchers in this field. Experimental verification of the coincidence effect was conducted in 1956 by Lamb, ${ }^{9}$ and then

\footnotetext{
${ }^{a)}$ Present address: Los Alamos National Laboratory, Geophysics Group, MS D443, Los Alamos, NM 87545. Electronic mail: bea@lanl.gov.
}

also in 1957 by Eichler and Lambert. ${ }^{10}$ Numerous studies have been conducted on the transmission of sound through partitions, but generally these studies are either done for subcritical plates or they measure the diffuse field transmission characteristics (an average over all angles of incidence, generally by using a reverberation chamber).

It was not until Davies and Gibbs' work in 1981 that a practical technique for measuring the angular dependence of sound transmission through a partition was proposed. ${ }^{11}$ Davies and Gibbs used a method similar to that proposed by Louden $^{12}$ where an impulsive source sends sound through the partition under test at selected angles. The direct arrival of energy through a partition was compared to the direct arrival of energy received without the partition present, thus allowing the sound transmission to be characterized. They utilized various averaging schemes to improve upon their results. Unfortunately, their technique was contaminated by spherically incident wave fronts which excited bending waves in the partition which produced arrivals of energy shortly after the direct arrival (which was later termed the precursor phenomenon). Balilah and Gibbs further refined the technique proposed by Davies and Gibbs by making the technique more portable and by suggesting ways to reduce the effect of the precursor phenomenon. ${ }^{13}$ Unfortunately these techniques require a large area of the material under test (partition) to avoid the arrival of the flanking transmission around the partition, thus smaller samples of material cannot be tested.

This paper proposes a technique in which a pulsed wave field is incident upon a plate which has an array of transducers mounted on the back side of the plate. The incident signal is emitted by a transmitter which is located in the far-field of the array. The measurement is repeated for many angles in the forward $180^{\circ}$. The signals detected by the array of transducers can be digitally steered in postprocessing to provide a 
high degree of directivity in the direction of the incident wave energy. Thus the problem of incident spherical waves is avoided. The wave field detected from the same array without a plate is also measured. A comparison of the detected signals with and without the plate yields the transmission as a function of angle of incidence.

In this paper a comparison of theoretical analysis to experimental results is given for the angular dependence of sound transmission through a plate. The theoretical analysis utilizes an expression derived from unbounded/infinite plate theory (finite thickness). Experimental results were obtained with a finite sized plate. A more complete discussion of the theory, modeling, and experiments conducted in this study are given in the dissertation by Anderson. ${ }^{14}$

\section{TRANSMISSION THROUGH UNBOUNDED FLEXIBLE PARTITIONS}

This section will provide a brief presentation of the theory of sound transmission through an unbounded flexible partition vibrating in flexure (infinite-sized, thin-plate theory). A complete theoretical analysis of this theory is given by Fahy (Ref. 15, pp. 277-295). Theory for unbounded flexible partitions can also be used as an approximation for modeling the angular dependence of sound transmission for finite sized plates.

The specific acoustic impedance, $\widetilde{z}_{\mathrm{SP}}$, for an unbounded flexible plate partition is given by

$$
\tilde{z}_{\mathrm{SP}}=\frac{1}{\omega} D \eta \kappa^{4}-j \frac{1}{\omega}\left(D \kappa^{4}-m \omega^{2}\right),
$$

where $\omega$ is the angular frequency, $D$ is the bending stiffness of the plate, $\eta$ is the damping loss factor in the plate, $\kappa$ is the structural wave number in the plate, $j$ is the imaginary number $\sqrt{-1}$, and $m$ is the mass per unit area of the plate. The structural wave number in the plate depends on the wave speed of the type of wave excited. Two types of waves will be considered here, driven waves excited by an incident wave field, and bending waves which have dispersive wave speeds which increase as a function of $\sqrt{\omega}$ for low frequencies (at larger frequencies the bending wave speed asymptotically approaches the shear wave speed).

The general transmission coefficient, $\tau$, for sound transmission through an unbounded flexible partition is given in Fahy's Eq. (5.36) (with some additional substitutions) as

$$
\tau=\left|\frac{2 \rho_{2} c_{2} \sec \theta_{2}}{\frac{1}{\omega} D \eta \kappa^{4}-j \frac{1}{\omega}\left(D \kappa^{4}-m \omega^{2}\right)+\rho_{1} c_{1} \sec \theta_{1}+\rho_{2} c_{2} \sec \theta_{2}}\right|^{2} \frac{\rho_{1} c_{1}}{\rho_{2} c_{2}}\left[\frac{1-\left(\frac{c_{2}}{c_{1}} \sin \theta_{1}\right)^{2}}{1-\sin ^{2} \theta_{1}}\right]^{1 / 2},
$$

where $\theta$ is an angle measured from normal incidence, $\rho$ is the density, $c$ is the acoustic speed of sound, and the subscripts 1 and 2 correspond to material properties of the incident and transmitted acoustic fluids (or the corresponding angle on either side of the partition). The term in the square brackets of Eq. (2) governs refraction of a wave from one fluid to the other, and if the fluid on both sides is the same then the refraction term and the impedance ratio term vanish and Eq. (2) becomes

$$
\tau=\frac{(2 \rho c \sec \theta)^{2}}{\left[2 \rho c \sec \theta+\frac{1}{\omega} D \eta k^{4} \sin ^{4} \theta\right]^{2}+\left[m \omega-\frac{1}{\omega} D k^{4} \sin ^{4} \theta\right]^{2}},
$$

where $k$ is the acoustic wave number $(k \sin \theta=\kappa)$. The second term in the denominator of Eq. (3) is governed by the damping in the plate, while the third and fourth terms are governed by the mass and stiffness in the plate, respectively. The standard equation for normal incidence mass law transmission results when Eq. (3) is evaluated at $0^{\circ}$, or when the bending stiffness term of Eq. (3) is very small compared to the mass term ( $\eta$ is generally much less than one).

The critical frequency, $f_{C}$, is defined as the frequency at which the acoustic sound speed in water equals the bending wave speed in the plate. The bending wave speed in a thin plate (thin plate theory ignores effects due to shear deformation and rotary inertia) is dispersive and increases with frequency,

$$
c_{B}=\left(\omega^{2} \frac{D}{m}\right)^{1 / 4} .
$$

At the critical frequency transmission through a plate at grazing incidence is very high. Above the critical frequency, as the frequency is increased, the angle of high transmission (called the coincidence angle, $\theta_{\mathrm{CO}}$, which occurs when the imaginary part of the plate's impedance goes to zero) shifts toward normal incidence

$$
\sin ^{2} \theta_{\mathrm{CO}}=\frac{c^{2}}{c_{B}^{2}}=\frac{c^{2}}{\omega} \sqrt{\frac{m}{D}} .
$$

Thus, the coincidence angle is proportional to the arcsine of the inverse square root of frequency. The critical frequency may be solved for by evaluating Eq. (5) at grazing incidence $\left(\theta_{\mathrm{CO}}=90^{\circ}\right)$ 


$$
f_{C}=\frac{c^{2}}{2 \pi}\left(\frac{m}{D}\right)^{1 / 2}
$$

The transmission coefficient at the coincidence angle is

$$
\tau\left(\theta_{\mathrm{CO}}\right)=\left(1+\frac{\eta m \omega}{2 \rho c} \sqrt{1-\frac{c^{2}}{\omega} \sqrt{\frac{m}{D}}}\right)^{-2} .
$$

A steered array of transmitting transducers effectively approximates a traveling wave in the plane of the array. The speed of this traveling wave must exceed the speed of sound in the fluid medium if the radiation is to be between grazing and normal incidence. This principle is called trace wave number matching (Ref. 15, pp. 175-177). In order to radiate sound at a given forward angle, $\theta_{0}$, one must set up a traveling wave on a steered array such that

$$
c_{0}=\frac{c}{\sin \theta_{0}}=\frac{d}{\tau_{0}}
$$

where $c_{0}$ is the speed of the traveling wave, $d$ is the centerto-center distance between consecutive transducers in the array, and $\tau_{0}$ is the electronic time delay necessary to progressively implement across the array between consecutive transducers. Thus if one scans the surface of an array and measures the effective traveling wave sound speed, one can then determine at what angle the array is radiating.

\section{EXPERIMENT SETUP}

\section{A. Array setup}

Two different one-dimensional eight element arrays were mounted onto the polyurethane acoustic window of an array module. The transducers used are of a typical Tonpilz design with a nominal resonance frequency of $25 \mathrm{kHz}$. One array was placed directly on the acoustic window, while the other array was bonded onto an alumina bar with a $1.6-\mathrm{mm}$ thick layer of polyurethane between the array elements and the alumina bar. The alumina bar is a high purity $99.8 \%$ aluminum oxide ceramic (AmAlOx 68 alumina) and is $1.02 \mathrm{~cm}$ thick, $3.05 \mathrm{~cm}$ wide, and $24.4 \mathrm{~cm}$ in length. Plastic shims were placed between neighboring transducers to maintain a consistent spacing between all transducers. Figure 1(a) shows a photograph of a close up of the array bonded to the alumina bar. Figure 1(b) shows a photograph of the two arrays placed side by side on the window of the array module.

\section{B. Scanning laser measurements}

To confirm that the appropriate traveling flexural waves are generated in the plate driven by the array of transducers, a set of measurements was conducted using a PolyTec scanning laser vibrometer. The vibration of the plate induced by the array was imaged with the scanning laser. Reflective tape was placed on the acoustic window (assumed to be acoustically transparent) to provide high reflectivity of the laser signal and to provide an accurate measure of the plate vibration. A 7 by 43 point scan grid was used with a spacing of $6 \mathrm{~mm}$ between scan points. The measurements were conducted with the array module submerged in a small water tank with an optically transparent wall to allow the laser to scan the
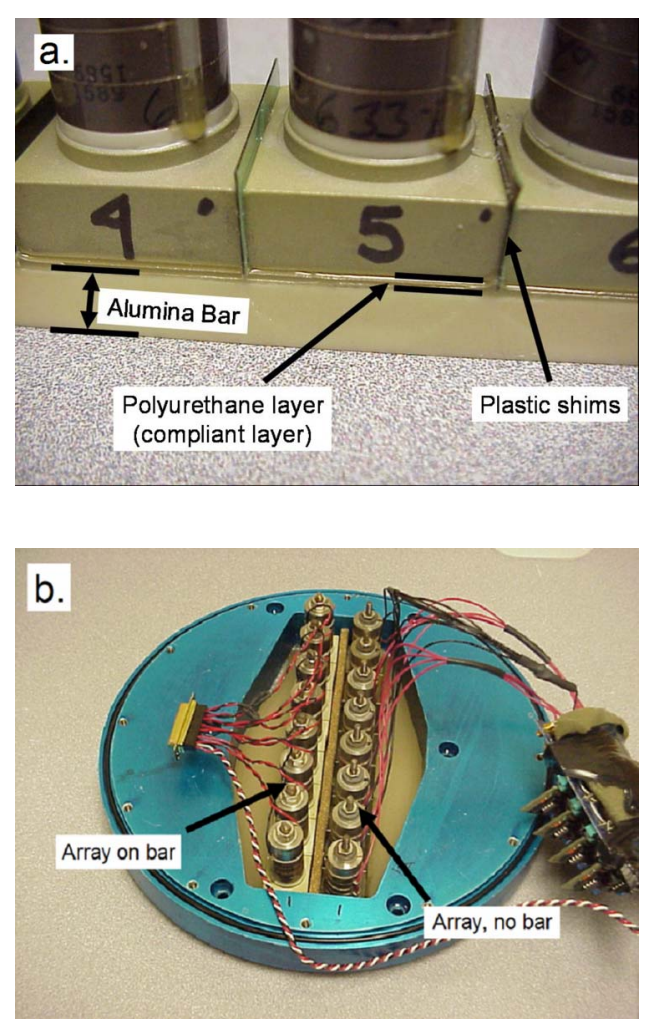

FIG. 1. (Color online) (a) Close up photograph of the alumina bar with an eight element line array mounted on it. (b) Photograph of the two eight element line arrays used in the water tank measurements.

submerged surface while being physically located outside the water. Figure 2(b) shows a photograph of the scanning laser measurement setup. The array was operated in transmit mode at various frequencies and steer angles. Care was taken to eliminate the possibility of obstruction of the desired data from a reflected wave off the tank walls by applying an appropriate time window and transmit repetition rate.

\section{Water tank measurements}

The water tank facility at Penn State measures 5.3 $\times 5.5 \times 7.9 \mathrm{~m}$ in size. The water tank is kept at a constant temperature and is lined with acoustical absorbing material. A calibrated transmitter or a calibrated receiver can be placed at one end of the tank to transmit/receive sound toward/from a transducer array under test at the other end of the tank. A computer controlled turntable can rotate the Array Under Test (AUT) with fractional degree increments. When the AUT is in a receive mode, up to 64 channels of complex data may be simultaneously acquired by a vector signal analyzer. The advantage of receive mode measurements is that in the postprocessing phase, the array can be arbitrarily steered to any angle by applying appropriate digital delays. In transmit mode each channel of the AUT must be amplified separately and set beforehand with fixed digital delays. Figure 2(a) shows a photograph of the array module just before being lowered into the water tank.

The array module was lowered into the water and connected up to the turntable. In one set of measurements the array mounted on the alumina bar was operated in a transmit 

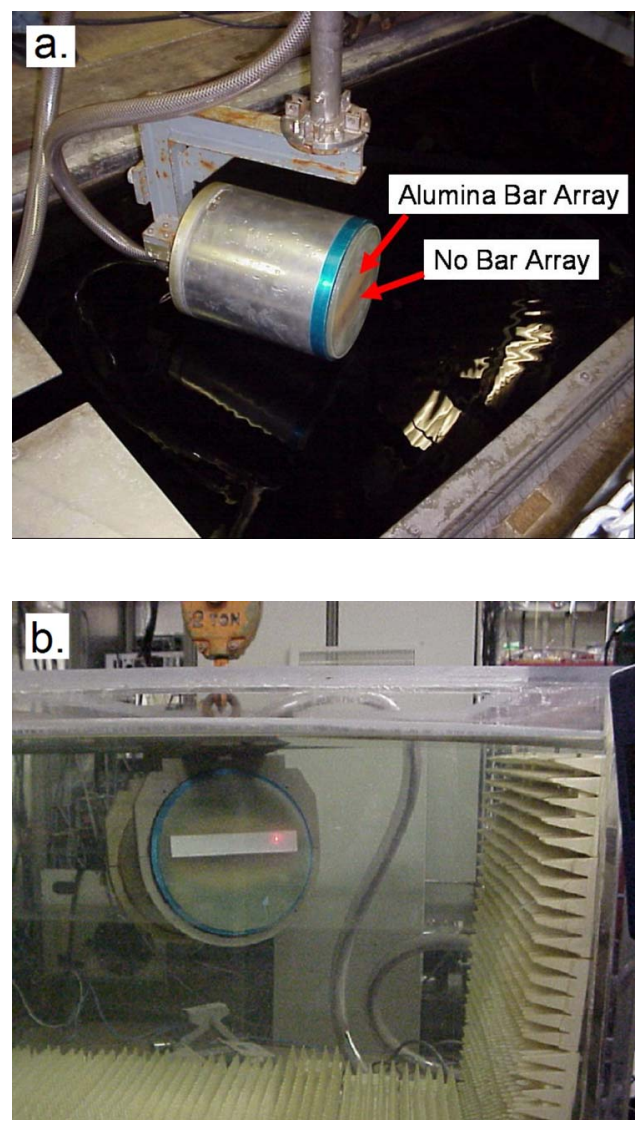

FIG. 2. (Color online) (a) Photograph of the array module before being lowered into the water tank. (b) Photograph of the array module in the small water tank while performing a scanning laser measurement.

mode at various frequencies and also at various steer angles by applying appropriate digital delays. In another set of measurements the array mounted on the bar and the array without a bar were each operated in a receive mode at various frequencies while recording complex data on each receiver channel.

\section{RESULTS}

\section{A. Scanning laser measurements}

The scanning laser results for a steer angle of $15^{\circ}$ at a frequency of $25 \mathrm{kHz}$ may be found in Fig. 3. The results are given at four different phase snapshots [(a) $0^{\circ}$, (b) $80^{\circ}$, (c) $180^{\circ}$, and (d) $\left.280^{\circ}\right]$ to illustrate the propagation of the traveling wave from right to left from Figs. 3(a)-3(d). According to Eq. (8), the speed of the steered array traveling wave, $c_{S}$, should approximately equal $1500 \mathrm{~m} / \mathrm{s} / \sin \left(15^{\circ}\right)=5800 \mathrm{~m} / \mathrm{s}$. In Fig. 3(a), the peak on the right-hand side travels about 29 scan points $(6 \mathrm{~mm}$ between points, therefore $174 \mathrm{~mm})$ to its location in Fig. 3(d) in $280^{\circ}$ of the phase cycle. That means that for a full $360^{\circ}$ cycle, the wave travels $224 \mathrm{~mm}$. The time duration, or period, of a cycle corresponds to the inverse of the frequency. Thus the duration of a period at $25 \mathrm{kHz}$ is $40 \mu \mathrm{s}$. The traveling wave speed then is $224 \mathrm{~mm} / 40 \mu \mathrm{s}$ $=5600 \mathrm{~m} / \mathrm{s}$. This value represents a fairly rough estimation, but it is within $3.5 \%$ of the $5800 \mathrm{~m} / \mathrm{s}$ expected sound speed. Thus the scanning laser results show that the dominant trav-
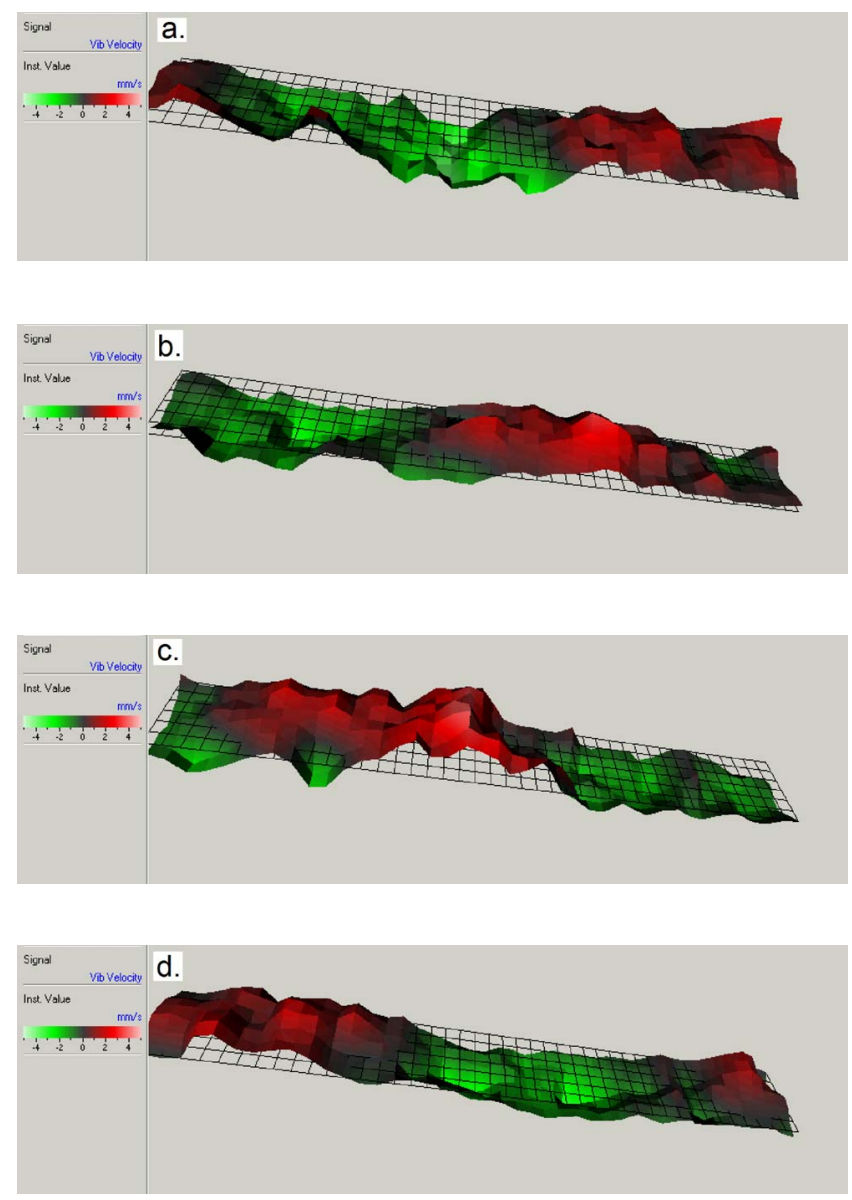

FIG. 3. (Color online) Velocity data from scanning laser data at $25 \mathrm{kHz}$ and $15^{\circ}$. Each subplot represents progressive phase snapshots $\left[(\mathrm{a}) 0^{\circ}\right.$, (b) $80^{\circ}$, (c) $180^{\circ}$, and (d) $\left.280^{\circ}\right]$ of the traveling wave progressing from right to left.

eling flexural wave on the radiating surface of the bar corresponds to the intended traveling wave setup by the transducer array.

\section{B. Water tank measurements}

Figure 4 displays sample plots comparing directivity patterns, normalized to their respective main lobe peak levels, from the transmit measurements and the receive measurements with the alumina bar array. The near perfect agreement of transmit and receive directivity patterns demonstrates that the array mounted on the alumina bar is a reciprocal system (Ref. 16, p. 378). The measurements of patterns from the array operated in a receive mode show that the data taken at large angles (approximately $> \pm 60^{\circ}$ ) is corrupted by the noise floor of the system.

With array measurements in receive mode, the data may be processed to compute directivity patterns steered to any forward angular direction desired. Thus a $180^{\circ}$ directivity pattern may be generated for each of the available $180^{\circ}$ of steer angles. One convenient method of displaying these data is to create an amplitude map surface plot of receive angle versus steer angle, such that a vertical line (from $-90^{\circ}$ to $+90^{\circ}$ ) at a fixed steer angle corresponds to the directivity pattern at that steer angle. Figure 5 displays sample surface plot maps of amplitude versus receive angle versus steer 

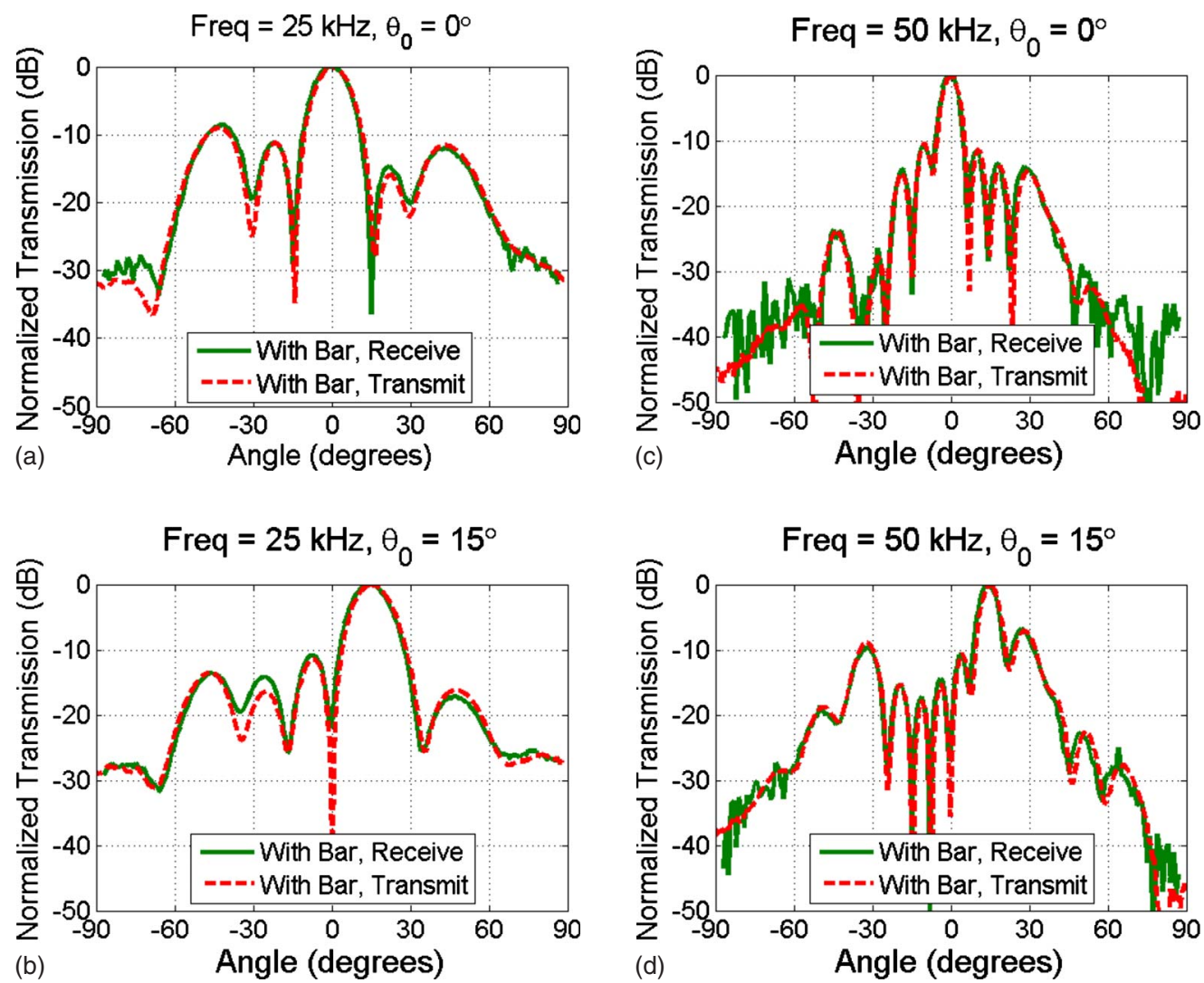

FIG. 4. (Color online) Directivity pattern plots of amplitude vs receive angle for the array mounted on the alumina bar operated in a transmit mode and in a receive mode at the frequencies and steer angles displayed in the titles of each subplot.

angle, with each vertical line of data normalized to its respective main lobe level, at $25 \mathrm{kHz}$ (left subplots) and $50 \mathrm{kHz}$ (right subplots) and for the array without the bar (upper subplots) and the array with the alumina bar (lower subplots). The dominant ridge in each subplot running from the lower left to the upper right corresponds to the main lobe at each steer angle. The presence of this ridge in the surface plots from the data taken from the array mounted on the bar demonstrates that the array can in fact steer sound energy through the alumina bar at the desired steer angle without refraction. Thus it is accurate to assume that the refraction term in square brackets in Eq. (2) may be discarded. Note that the width of the main lobe ridge in data taken from each of the two arrays is approximately the same, meaning that the presence of the alumina bar does not widen the main lobe. Thus the dispersive nature of bending waves in the bar does not alter the driven wave speed, since widening of a lobe would correspond to dispersion of wave speeds. The absence of refraction and dispersion, in the transmission of the driven wave through a plate, are important practical considerations when evaluating the feasibility of using an array to image objects behind a plate.

Note the horizontal ridges which occur in Figs. 5(b) and 5 (d) located at receive angles of $\pm 45^{\circ}$ and at $\pm 30^{\circ}$, respectively. These horizontal ridges are located at the respective coincidence angles for each frequency [from Eq. (5), $\theta_{\mathrm{CO}}(25 \mathrm{kHz})=45^{\circ}$ and $\theta_{\mathrm{CO}}(50 \mathrm{kHz})=30^{\circ}$ for the alumina bar used]. The coincidence angle is the angle at which bending waves radiate most efficiently.
Another way to analyze the effect of the presence of the alumina bar is to compare the level of the main lobe at each steer angle from the data taken from each array configuration (with and without the alumina bar). The only difference between these two sets of steering data is due to the alumina bar, thus it provides a convenient method of measuring the sound transmission through the alumina bar as a function of angle. The theoretical expression for sound transmission through an undamped $(\eta=0)$ plate or bar given in Eq. (3) was evaluated at various frequencies using material property values which correspond to measured values for the alumina bar density $\left(\rho=3956 \mathrm{~kg} / \mathrm{m}^{3}\right)$, Young's modulus $(E$ $=391 \mathrm{GPa})$, and bar thickness $(h=1.02 \mathrm{~cm})$. [Thus the critical frequency for the alumina bar is $f_{C}=12.0 \mathrm{kHz}$, assuming the Poisson ratio specified by the manufacturer $(\sigma=0.22)]$.

Figure 6 shows a comparison of the theoretical and measured sound transmission through an alumina bar. Both plots are normalized to the normal incidence mass law sound transmission levels (measured plots were adjusted such that the small increase at normal incidence was ignored). The theoretical expressions were not evaluated at angles greater than $\pm 65^{\circ}$ since the theoretical expression does not generally give accurate results at large angles, as noted by Fahy (Ref. 15, pp. 292-294). Also, measurements at large angles are corrupted by the measurement noise floor as noted in the reciprocity comparison discussion. Although the analytic approach is approximate (based on thin infinite plate theory), the simulated general behavior matches that observed in the 
Theory, No Bar, @ 25 kHz
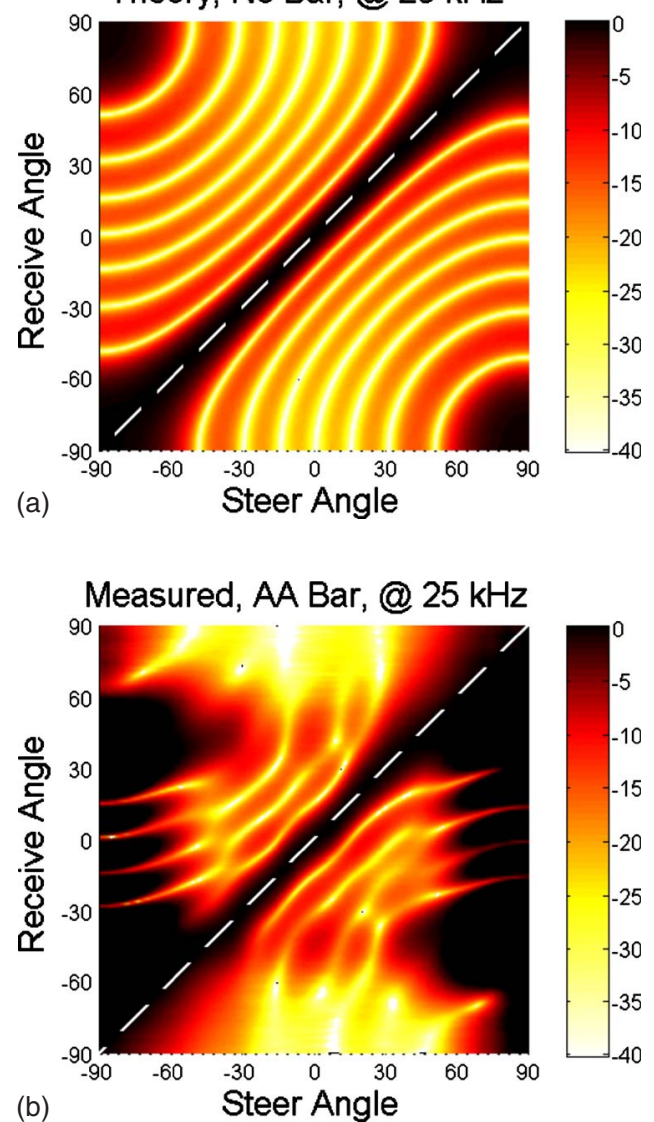

Theory, No Bar, @ 50 kHz

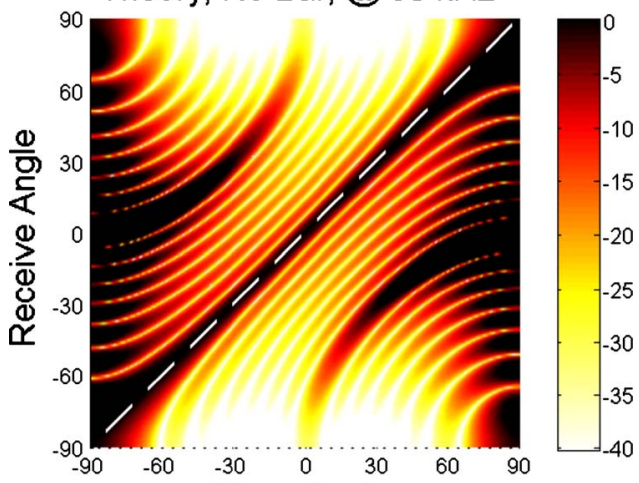

(c)

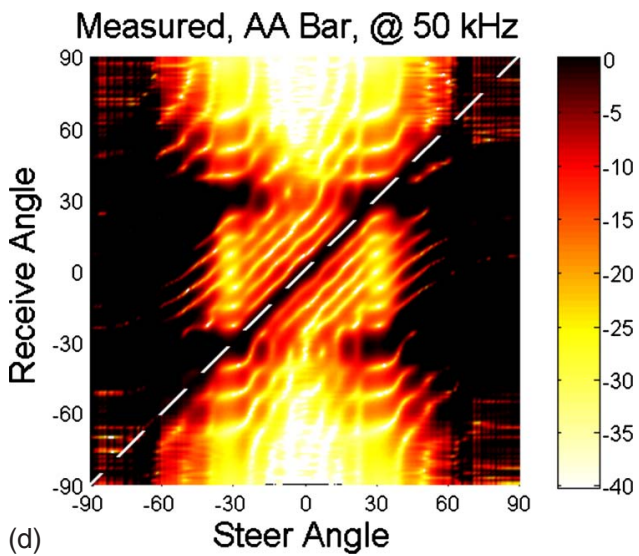

FIG. 5. (Color online) Surface plots of directivity amplitude vs steer angle and receive angle. The upper subplots are from data without the alumina bar, while the bottom subplots are from data with the alumina bar. The subplots on the left are for $25 \mathrm{kHz}$ while the subplots on the right are for $50 \mathrm{kHz}$

measurements. This means that the approximate analytic theory may be used for qualitative studies of other plate and array configurations. The discrepancies in levels at coincidence angles and at normal incidence are due to the approximate nature of the analytics used here (infinite, rather than finite plate; thin plate theory, rather than thick; approximate treatment of damping).

\section{CONCLUSION}

Results have shown that indeed a phased array of transducers can induce a traveling flexural wave on a rectangular bar which then radiates sound into the same direction as the phased array would radiate without a bar. Results also show that placing a bar in the near-field of the array allows measurement of transmission versus angle. The measured transmission versus angle for a finite sized plate does agree qualitatively with the theoretical transmission versus angle for an unbounded thin plate of the same thickness and material properties. It should be noted that the frequencies used in this study were above the plate's critical frequency. Below the critical frequency, one would not encounter the coincidence effects in the array's radiation pattern, since bending waves would not radiate efficiently even though they would be excited.

The ability to steer sound energy through a plate allows one to image objects on the other side of the plate when it is otherwise impossible to physically access that side of the plate. Sound transmission through a partition or plate is the highest at the coincidence angle, thus applications could be developed to exploit the ability to transmit and receive sound with a high degree of transmission at this angle. Conceivably, an array may be placed on the wall of a submarine hull or boat to image objects in the water.

A novel technique which allows measurement of the transmission coefficient as a function of angle has been presented in this paper. This technique is similar in nature to the techniques proposed by Louden, ${ }^{12}$ Davies and Gibbs, ${ }^{11}$ and Balilah and Gibbs ${ }^{13}$ in that sound is sent through a plate at various angles, thus allowing measurement of the angular dependent transmission coefficient. However, the advantage of the proposed technique is that it exploits the high directivity of an array to avoid the flanking transmission and precursor waves encountered in the research done by Davies and Gibbs and Balilah and Gibbs which were due to using spherical wave sources. In addition, while the techniques proposed by the above-mentioned researchers essentially require relatively large partition samples, the proposed technique measures relatively small plate samples.

The results presented here were for one-dimensional measurements of the angular dependent transmission coefficient using a line array and a bar under test. The investigation has been extended to two dimensions by the authors, using a two-dimensional array of transducers. ${ }^{14}$ The measurements yielded results consistent with the results found in 

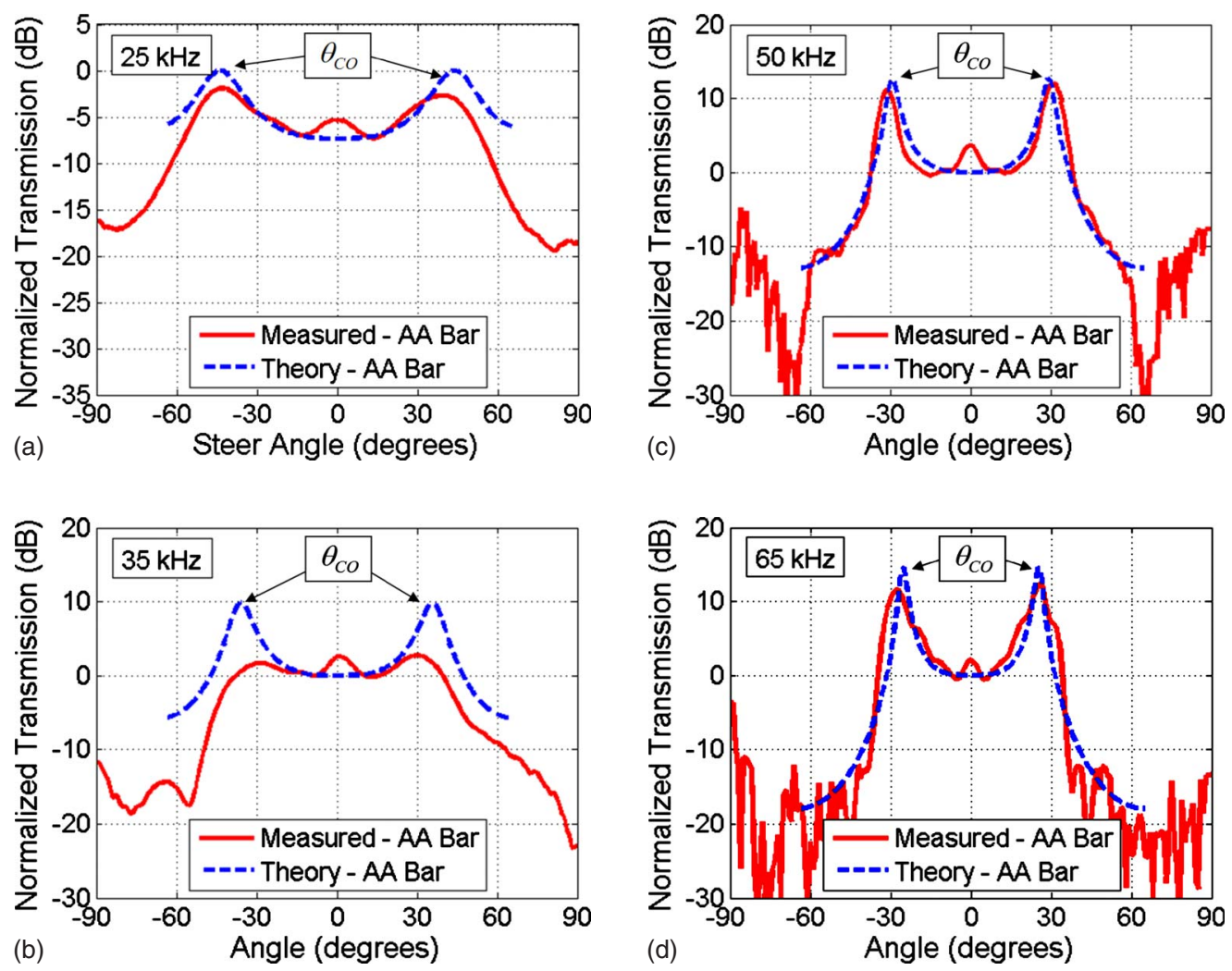

FIG. 6. (Color online) Plots of transmission through the alumina (AA) bar vs incidence angle for the frequencies indicated in the upper left of each subplot.

the material presented in this paper, and may be presented in a future paper. Finally, the analytic approach presented here (based on infinite thin plate theory) is approximate. Future analytic work in this area should focus on thick plate effects, and on finite dimensions, perhaps using numerical methods.

\section{ACKNOWLEDGMENTS}

This research was sponsored by the Office of Naval Research Code 333 Dr. David Drumheller. In addition to ONR support for B.A., support was also provided by a Penn State University Graduate Research Fellowship, a Penn State College of Engineering Fellowship, an Audio Engineering Society Educational Foundation Award, and Penn State Applied Research Laboratory Exploratory and Foundational Research Program funding. The assistance of Paul Bednarchik, Courtney Burroughs, Bob Dashem, Mark Geleskie, Greg Granville, Bill Thomas, Dave Van Tol, and Mark Wilson of the Penn State Applied Research Laboratory is recognized and appreciated. Facilities located in the Applied Research Laboratory of The Pennsylvania State University were used in this work. Electronic mail correspondence with Frank Fahy was also quite helpful in this work.

${ }^{1}$ J. S. Hickman, D. E. Risty, and E. S. Stewart, "Properties of sandwichtype structures as acoustic windows," J. Acoust. Soc. Am. 29, 858-864 (1957).

${ }^{2}$ E. E. Mikeska and J. A. Behrens, "Evaluation of transducer window ma- terials," J. Acoust. Soc. Am. 59, 1294-1298 (1976).

${ }^{3}$ D. L. Folds and C. D. Loggins, "Transmission and reflection of ultrasonic waves in layered media,” J. Acoust. Soc. Am. 62, 1102-1109 (1977).

${ }^{4} \mathrm{C}$. M. Thompson, "Development of a structurally rigid, acoustically transparent plastic," J. Acoust. Soc. Am. 87, 1138-1143 (1990).

${ }^{5}$ M. Kim and Y. F. Hwang, "An analysis of wave dispersion in coarsely laminated symmetric composite plates,” J. Acoust. Soc. Am. 100, 19811991 (1996).

${ }^{6}$ J. I. R. Blake, R. A. Shenoi, J. House, and T. Turton, "Strength modeling in stiffened FRP structures with viscoelastic inserts for ocean structures," Ocean Eng. 29, 849-869 (2002).

${ }^{7}$ A. J. Hull, "Dynamic response of an insonified sonar window interacting with a Tonpilz transducer array," J. Acoust. Soc. Am. 122, 794-803 (2007).

${ }^{8}$ L. Cremer, "Theorie der Schall damming dunner Wade bei Schragen Einfall," Akust. Z. 7, 81-104 (1942).

${ }^{9}$ G. Lamb, Jr., "Transmission of a spherical wave through a thin elastic plate," J. Acoust. Soc. Am. 28, 162 (1956).

${ }^{10}$ E. G. Eichler and R. F. Lambert, "Sound Transmission through thin plates at oblique incidence," J. Acoust. Soc. Am. 29, 1253 (1957).

${ }^{11}$ J. C. Davies and B. M. Gibbs, "Oblique incidence measurement of transmission loss by an impulse method," J. Sound Vib. 74, 381-393 (1981).

${ }^{12}$ M. M. Louden, "The single-pulse method for measuring the transmission characteristics of acoustic systems," Acustica 25, 167-172 (1971).

${ }^{13}$ Y. A. Balilah and B. M. Gibbs, "The measurement of the transmission loss of single leaf walls and panels by an impulse method," J. Sound Vib. 123, 229-245 (1988).

${ }^{14}$ B. E. Anderson, "Grating lobe reduction in transducer arrays through structural filtering of supercritical plates," Ph.D. thesis, The Pennsylvania State University, University Park, PA.

${ }^{15}$ F. Fahy and P. Gardonio, Sound and Structural Vibration, Radiation, Transmission and Response, 2nd Ed. (Academic Press, London, 2007).

${ }^{16}$ L. L. Beranek, Acoustics (McGraw-Hill, New York, 1954). 